\title{
Delayed recognition of esophageal intubation
}

\author{
Kenneth C. Dittrich, MD
}

\begin{abstract}
It is important for clinicians to be aware of the sensitivity and limitations of commonly used methods to confirm endotracheal tube placement. Overreliance on insensitive indicators can lead to delayed recognition of esophageal intubation. The case presented highlights this concern.

RÉSUMÉ

Il est important pour les cliniciens de connaître la sensibilité et les limites des méthodes couramment utilisées pour confirmer la position de la sonde endotrachéale. Une trop grande confiance accordée à des indicateurs insensibles peut conduire à une identification retardée d'une intubation oesophagienne. Le cas présenté dans cet article met en lumière une telle préoccupation.
\end{abstract}

Key words: esophageal, intubation, mortality

\section{Introduction}

Ensuring proper endotracheal tube (ETT) placement is paramount in emergency airway management. In attempting to acquire a definitive airway, it is not uncommon to inadvertently intubate the esophagus. Clinicians rely upon various signs and techniques to recognize this error, thereby preventing serious morbidity or mortality. These methods are well described in the literature; however, it is important for clinicians to adhere to these confirmatory tests and to recognize their limitations. The case presented is that of delayed recognition of an esophageal intubation resulting in the demise of the patient. This case helps to identify common misconceptions and pitfalls in verifying ETT placement.

\section{Case report}

A 6-year-old male was brought to the emergency department (ED) 30 minutes after an accidental gunshot wound to the abdomen (.44 calibre hand gun). On arrival the patient was conscious and alert, with blood pressure 130/70 mm
$\mathrm{Hg}$, pulse 140 beats/min and respiratory rate 32 breaths/min. Pulse oximetry measured oxygen saturation at $88 \%$ initially on room air. Lung fields were clear on examination, and capillary refill was less than 2 seconds. $100 \%$ oxygen by a non-rebreathing mask was applied. Two 18-gauge intravenous lines with normal saline were inserted. The presence of tachycardia and relative hypertension raised the suspicion of early shock, and a $20 \mathrm{cc} / \mathrm{kg}$ fluid bolus was initiated. Entry and exit wounds were identified at the left elbow, and a re-entry wound was present at the left ninth intercostals space, mid-axillary line. The .44 calibre missile was palpable sub-cutaneously in the right flank at the mid-axillary line. A supine chest/abdominal x-ray was taken. The thoracic film (Fig. 1) was normal, without evidence of pneumothorax. The abdominal film identified the missile in the right flank. A nasogastric tube was inserted, with position confirmed by epigastric auscultation following air insufflation. Appropriate blood work and cross match were sent to the lab. The surgeon on duty and the operating room (OR) staff were notified immediately of the case.

Despite being on supplemental oxygen, saturations were noted to be falling, to between $77 \%$ and $80 \%$, during the 
first 15 minutes since arrival. The patient's hemodynamic status remained unchanged. A decision was made to insert a prophylactic left thoracostomy tube and intubate the patient prior to going to the OR. Rapid sequence intubation (RSI) was initiated using atropine and fentanyl followed by midazolam and succinylcholine. The ETT was perceived by the operator to have been passed into the trachea by direct vision. Auscultation of both axilla noted "good air entry," and condensation of water vapour in the tube was also noted. Neither an end tidal $\mathrm{CO}_{2}$ monitor nor an esophageal detector device were used. An arterial blood gas sample drawn during the pre-oxygenation phase of the RSI showed that the $\mathrm{PO}_{2}$ was $286 \mathrm{~mm} \mathrm{Hg}$ with $99.6 \%$ oxygen

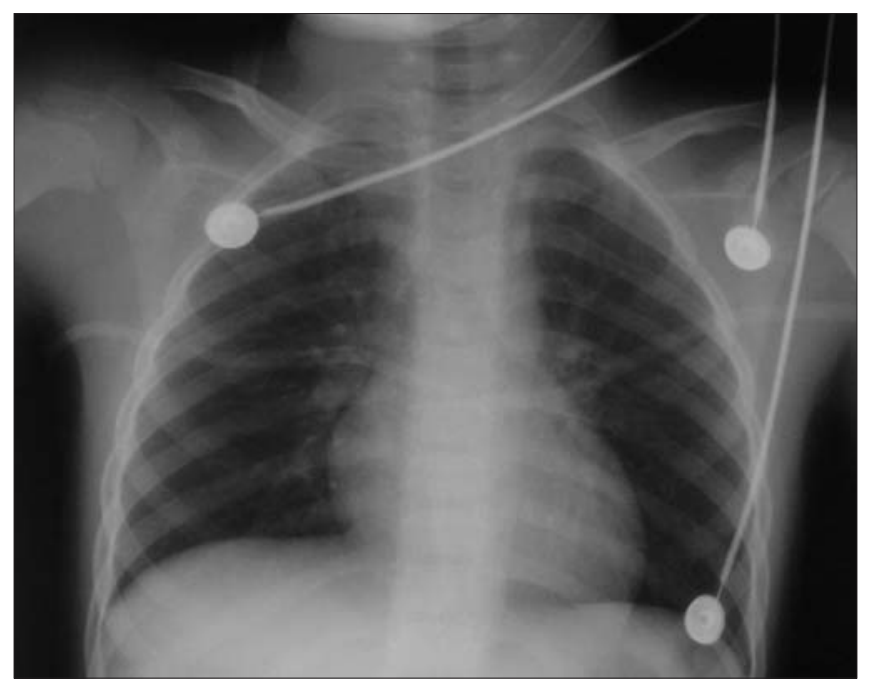

Fig. 1. Initial chest x-ray

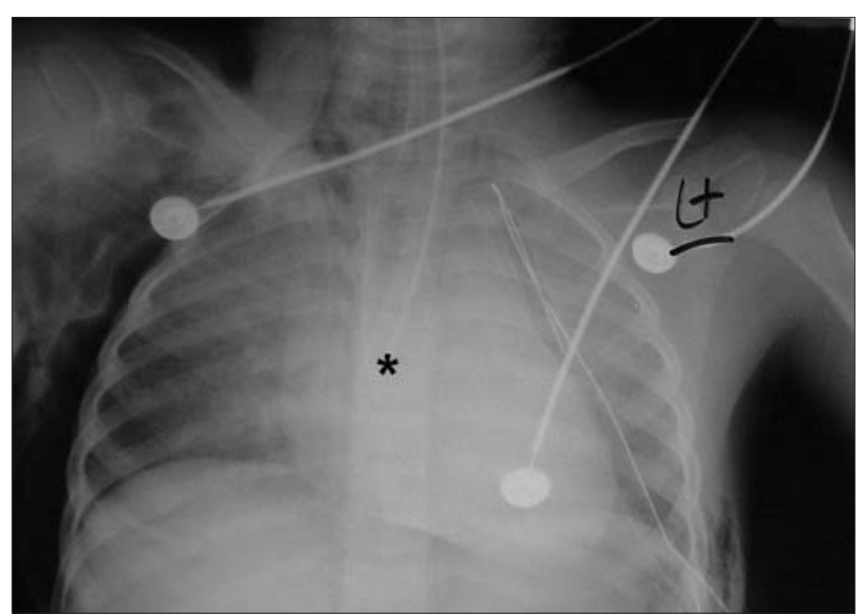

Fig. 2. Chest x-ray post esophageal intubation. The distal tip of the endotracheal tube (asterix) lies below the take-off of the right mainstem bronchus. In comparison with Fig. 1, decreased aeration of both lung fields is also noted. NG tube distal tip is at the cardia of the stomach. Left sided thoracostomy tube is kinked. saturation. Follow-up oxygen saturation was not documented. Blood was suctioned from the ETT shortly after intubation. A repeat chest/abdominal x-ray (Figs. 2 and 3) was taken, and the patient was transferred directly to the OR. The x-ray film was not viewed by the attending physician prior to sending the patient to the OR, due to the immediacy of the transfer.

Upon arrival at the OR, the patient was found to have pulseless electrical activity, with a heart rate of 40 beats/min. A grossly distended abdomen was noted. Surgical emphysema was present across the abdomen, thorax and neck. Immediate assessment of the airway revealed an esophageal intubation. Airway edema and anatomic distortion secondary to surgical emphysema prevented endotracheal intubation, and a tracheostomy was performed. The patient's rhythm rapidly deteriorated to asystole and, despite all efforts, resuscitation was unsuccessful.

The official x-ray reading the following day erroneously reported the ETT to be in the right main stem bronchus. Later, upon case review and with the benefit of clinical information as to the discovery made in the OR, a second reading of the film identified clear indicators of ETT misplacement and the presence of pneumoperitoneum (Fig. 3).

\section{Discussion}

Esophageal intubation (EI) is a common complication of definitive airway management. In a review of 610 ED intubations by Sakles and colleagues, ${ }^{2}$ the incidence of inadvertent esophageal tube placement was found to be $5.4 \%$.

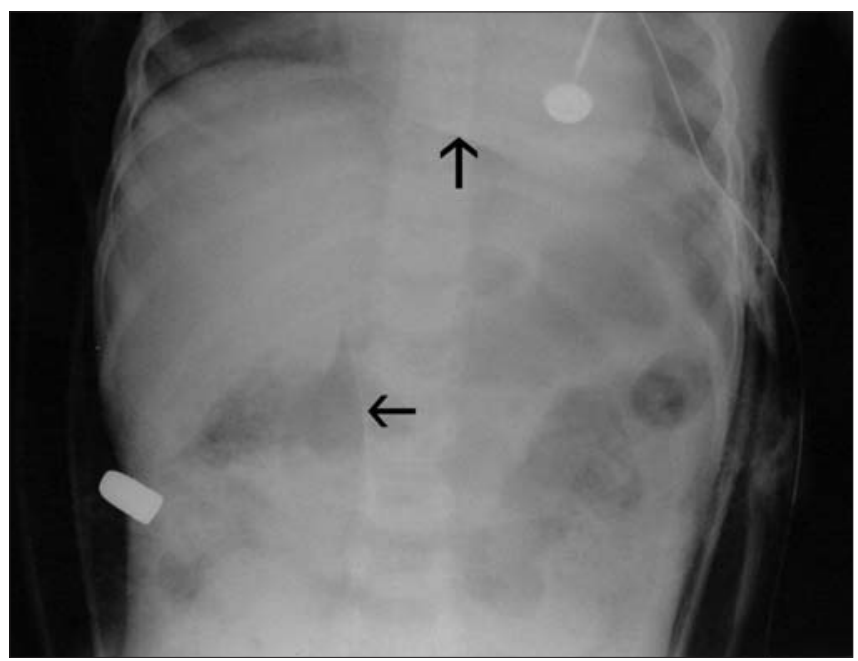

Fig. 3. Abdominal film. The .44 calibre missile is present in the right flank. Pneumoperitoneum is present creating a silhouette of ligamentum teres (horizontal arrow), and lower cardiac border (vertical arrow). ${ }^{2}$ Marked subcutaneous emphysema is also present. 
All were promptly recognized on clinical grounds and rapidly corrected. This rate is consistent with other studies. ${ }^{3,4}$ It has been found that experience alone does not prevent the occurrence of EI. Consultant physicians and trainees both have a similar incidence. ${ }^{5}$ Esophageal misplacement was also found to be as common in seemingly straightforward as well as difficult intubations. ${ }^{5} \mathrm{EI}$ is considered to be a problem of recognition as opposed to prevention. ${ }^{6}$ Timely assessment of proper ETT placement is necessary to avoid serious morbidity and mortality. Unfortunately there are inherent limitations to the common methods used to verify tube placement. Many physicians rely solely on these error-prone clinical detection methods, unaware of their insensitivity.

Procedures to evaluate tube position must be simple, reliable, inexpensive and rapidly applicable in order to achieve widespread acceptance. ${ }^{7}$ There are 2 direct confirmatory techniques of proper ETT placement: fiberoptic bronchoscopy with visualization of the tracheal rings through the ETT, and direct visualization of the endotracheal tube passing through the vocal cords. ${ }^{6}$ Bronchoscopy is not commonly available to the emergency physician, therefore the latter technique is the one more commonly felt to be the Gold Standard. ${ }^{6}$ Verification by direct visualization, although logically sound, is fraught with potential hazards. EI can occur even when the clinician believes he or she has observed the ETT pass through the larynx, as with this case. It may occur if the operator is distracted at the last moment before tube passage, she or he fails to accurately identify the anatomy, or the tube becomes displaced into the esophagus post-tracheal intubation. ${ }^{7}$ This may be problematic, in that a clinician convinced of proper tube placement will more likely explore other causes for hypoxia first, leading to a delayed recognition of the true cause.

Frequently, the sole method used by clinicians to verify proper tube placement is auscultation of the chest. Detecting the location of the ETT by this method may be inaccurate. Air flowing through the esophagus has been shown to easily mimic respiratory airflow. ${ }^{8}$ Auscultation of the axillary region alone has been found to be insensitive for misplaced ETT. Andersen and coworkers demonstrated this method failed to identify esophageal intubation in 5 of 40 cases. $^{8}$ Auscultation of the chest and the epigastrium improved sensitivity, but still failed to identify 1 of 40 esophageal intubations. ${ }^{8,9}$ Clearly auscultation alone should never be relied upon as the sole method of verification.

Another commonly used method is observing condensation of water vapour during expiration. Haridas, however, reported 42 of 60 esophageal intubations showing condensation..$^{10}$ It is well documented that condensation can occur from gas emanating from the stomach. ${ }^{7,910}$ This observation has proven to be unreliable, even as a supplementary sign.

Pulse oximetry should be continuously monitored during the intubation process. Its use as a rapid detector of EI is limited. The standard practice of pre-oxygenation prior to intubation delays desaturation. Delayed desaturation may lead the clinician to not associate this with a misplaced tube.

The chest $\mathrm{x}$-ray should not be utilized as a confirmatory test to differentiate esophageal from endotracheal tube placement. In addition to the normal delay incurred in taking and developing the film, misreading the $\mathrm{x}$-ray is possible due to projection of the esophagus over the tracheal air column. ${ }^{11}$ The utility of the chest $\mathrm{x}$-ray is primarily to ensure proper tube position within the trachea. Positive pressure ventilation in the presence of esophageal intubation has been reported to cause gastric rupture and pneumoperitoneum. ${ }^{12}$ The development of massive pneumoperitoneum in our case was attributed to hollow viscus perforation by the missile, along with gastric insufflation following misplacement of the ETT.

Over the last 2 decades capnometry (end-tidal $\mathrm{CO}_{2}$ monitoring) has been used as a reliable test to distinguish tracheal from esophageal intubation. ${ }^{13}$ Alveolar gas contains $5 \%$ carbon dioxide providing pulmonary perfusion is adequate. ${ }^{7}$ False positive readings, reporting the tube to be in the trachea when it is actually in the esophagus are possible. This may occur when expired alveolar gas is introduced into the stomach during bag-valve-mask ventilation, or following the ingestion of carbonated beverages or antacids. ${ }^{714}$ False positive readings reporting the ETT to be in the esophagus when it is actually in the trachea may occur in states of low cardiac output (e.g., cardiac arrest, severe hypotension), severe pulmonary disease or pulmonary embolism. ${ }^{14}$ In a meta-analysis by $\mathrm{Li},{ }^{14}$ capnography was shown to have a sensitivity of $93 \%$ and specificity of $97 \%$. This translates to a failed recognition of esophageal intubation in 3\% of cases. Qualitative colorimetric and quantitative digital end-tidal $\mathrm{CO}_{2}$ detector devices are available. The qualitative method simply measures the presence of $\mathrm{CO}_{2}$, whereas the quantitative method produces a waveform that can be correlated with the respiratory cycle. ${ }^{15} \mathrm{~A}$ detectable waveform can be identified at lower levels of expired $\mathrm{CO}_{2}$, making it more sensitive in low flow states. ${ }^{16}$ The presence of a waveform, no matter how small, provides strong evidence of endotracheal tube placement.

Esophageal detector devices (EDDs) rely on the differences in rigidity of the tracheal and esophageal walls. If the tube is in the esophagus there is resistance to aspiration. It is a rapid and reliable indicator of proper tube placement. Sensitivity and specificity have been reported 
to approach $100 \% .{ }^{17-19}$ Reports of failure of the EDD to detect esophageal intubation have been attributed to previous air insufflation into the stomach. ${ }^{20}$ False negative readings (i.e., failing to detect tracheal intubation) have been reported to occur in the setting of chronic obstructive pulmonary disease, copious secretions and obesity. ${ }^{21}$ The EDD has an advantage over capnometry in the overall ED population because of its greater sensitivity in the setting of cardiac arrest. ${ }^{21,22}$ In the pre-hospital setting, the EDD has been found to be less reliable. In a prospective study by Pelucio and colleagues of 168 patients intubated in the field by paramedics, the EDD failed to identify 5 out of 10 esophageal intubations. ${ }^{23}$ This further illustrates the need to use multiple confirmatory methods in order to detect tube misplacement. The EDD and the end-tidal $\mathrm{CO}_{2}$ monitor are both excellent but imperfect devices for determining tracheal versus esophageal tube placement. Even when endotracheal placement is confirmed, identifying right mainstem or ETT placement at the cords, can only be identified by chest radiography.

The case presented demonstrated the inaccuracy of commonly used clinical methods to confirm proper ETT placement. Direct visualization, auscultation and tube condensation were erroneously relied upon as sufficient and accurate markers. The official chest $\mathrm{x}$-ray reading as well reflected the difficulties in using this as a confirmatory test. Overreliance on these insensitive indicators led to an unnecessary position of risk that ultimately resulted in the patient's death. The end-tidal $\mathrm{CO}_{2}$ monitor and the EDD are the most reliable instruments routinely available to confirm proper tube placement. Both tests are highly sensitive and specific, as well as rapid, easy and inexpensive to use. Failure to use either may lead to an unrecognized esophageal intubation. Clinicians should utilize these objective tools in combination with clinical confirmatory measures to verify proper ETT placement.

Competing interests: None declared.

\section{References}

1. Cho KC. Visualization of the extrahepatic segment of the ligamentum teres: a sign of free air on plain radiographs. Radiology 1997;202:651-4.

2. Sakles JC, Laurin EG, Rantapaa AA, Panacek E. Airway management in the emergency department: A one-year study of 610 tracheal intubations. Ann Emerg Med 1998;31:325-32.

3. Schwartz DE, Mattay MA, Cohen NH. Death and other implications of emergency airway management in critically ill adults: a prospective investigation of 297 tracheal intubations. Anaesthesiology 1995;82:367-76.

4. Mort TC. Incidence and risks leading to cardiac arrest following emergency intubation [abstract]. Crit Care Med 1994;22:A137.

5. Holland R, Webb RK, Runciman WB. Oesophageal intubation: an analysis of 2000 incident reports. Anaesth Intensive Care 1993;21:608-10.

6. Wilson CW, Benumof JL. Respiration in anaesthesia pathophysiology and clinical update. Anesth Clin North Am 1998;16:29-75.

7. Clyburn P, Rosen M. Accidental oesophageal intubation. Br J Anaesth 1994;73:55-63.

8. Andersen KH, Schultz-Lebahn T. Oesophageal intubation can be undetected by auscultation of the chest. Acta Anaesthesiol Scand 1994;38:580-2.

9. Andersen KH, Registrar A, Hald A. Assessing the position of the tracheal tube. The reality of different methods. Anaesthesia 1989;44:984-5.

10. Haridas RP. Condensation on tracheal tubes is commonly seen with oesophageal intubation. Br J Anaesth 1995;75:115-6.

11. Smith GM, Reed JC, Choplin RH. Radiographic detection of esophageal malposition of endotracheal tubes. AJR 1990;154:23-6.

12. Ballester EE, Torres A, Rodriguez-Roisin R, Agusti-Vidal A. Pneumoperitoneum: an unusual manifestation of improper oral intubation. Crit Care Med 1985;13:138-9.

13. Ornato JP, Shipley JP, Racht EM, et al. Multicenter study of a portable, hand size, calorimetric end-tidal carbon dioxide detection device. Ann Emerg Med 1992;21:518-23.

14. Li J. Capnography alone is imperfect for endotracheal tube placement confirmation during emergency intubation. J Emerg Med 2001;20:223-9.

15. EasyCap $\mathrm{ETCO}_{2}$ Detector Product Information. Hayward (CA): Nellcor, Inc; 1992.

16. O'Connor RE, Swor RA. Verification of endotracheal tube placement following intubation. Prehosp Emerg Care 1999;3:248-50.

17. Wee MYK. The esophageal detector device: a rapid and accurate method for assessing tracheal versus esophageal intubation in the porcine model. Ann Emerg Med 1992;21:1073-6.

18. Williams KN, Nunn FJ. The esophageal detector device. Anaesthesia 1989;44:412-4.

19. Li J. A prospective multicenter trial testing the SCOTI device for confirmation of endotracheal tube placement. J Emerg Med 2001;20:231-9.

20. Andres AH, Langenstein $H$. The esophageal detector device is unreliable when the stomach has been ventilated. Anesthesiology 1999;91:566-8.

21. Kasper CL, Deem S. The self-inflating bulb to detect esophageal intubation during emergency airway management. Anaesthesiology 1998;88:898-902.

22. Bozeman WP, Hexter D, Liang HK, Kelen GD. Esophageal detector device versus detection of end-tidal carbon dioxide level in emergency intubation. Ann Emerg Med 1996;27:595-9.

23. Pelucio M, Halligan L, Dhindsa H. Out-of-hospital experience with the syringe esophageal detector device. Acad Emerg Med 1997;4:563-8.

Correspondence to: Dr. Kenneth Dittrich, Department of Emergency Medicine, King Fahad National Guard Hospital, Riyadh, Saudi Arabia; dittrich@naseej.com.sa 